\title{
Energy and entropy of metastable states in glassy systems
}

\author{
J. Christian Schön \\ Institut für Anorganische Chemie, Universität Bonn \\ Gerhard-Domagk-Str. 1, D-53121 Bonn \\ Paolo Sibani \\ Dept. of Physics, Odense Universitet \\ Campusvej 55, DK-5230 Odense $M$
}

(June 26, 2018)

\begin{abstract}
We investigate the multi-valley energy landscape of a 3-D on-lattice network model for covalent glasses, numerically determining the shape of the valleys, the local density of states, the density of minima and the local connectivity. We present some of these quantities in a graphical birds-eye view of the landscape, and discuss their implications for the relaxation dynamics and cooling behavior of glasses. The strong similarities between the landscape of this model and those of other complex systems point to the possibility of a common low-temperature dynamical description.
\end{abstract}

61.43.-j,61.43.Fs, 05.70.Fh

Introduction. A number of problems ranging from solid state physics to combinatorial optimization [1], through chemical physics 22, 5], and biology [6,7] can be formulated in terms of Marcov processes with states in the systems' energy landscapes. The (effective) connectivity of the landscape specifies the allowed hopping transitions while the energies determine their magnitude. Specifically for the multi-valley landscapes characterizing complex systems, local thermal equilibrium establishes itself within 'ergodic components' [8], and an investigation of the local geometry close to energy minima yields significant dynamical information.

For discrete systems, all configurations within subsets of the state space (pockets) centered around local energy minima can be counted 91. In addition, combinatorial techniques have been used to find all low-lying minima of small systems [10], and different approaches have been devised to coarse grain the configuration space into a space of more manageable size [4.5, 11 13 .

For clusters and glassy systems with continuous configuration spaces the main emphasis has been on stochastic methods for finding energy minima and the lowest saddles which connect them [2]. The vibrational modes of the systems can then be described within a harmonic approximation of the potential close to the minima.

Low temperature MC/MD-simulations have also been utilized to probe the vibrational and relaxational behaviour on short time scales in the neighborhood of "frozen-in-networks" 14 16. At higher temperatures, these methods yield insights into the dynamics of the glass transition and aging phenomena 17,18 . In addi- tion, the large scale distribution of local minima [19,20] can be sampled by quenching out of the melt.

While knowing the minima and their connecting saddles is useful in predicting e.g. whether a given system will be glass forming or not [3,5, 13, quantitative knowledge of the density of states of model glasses is still rather scanty. This information can be useful in clarifying the connection between structural glasses and systems with quenched randomness as e.g. spin-glasses and help to provide a unified picture of the energy landscapes of complex systems.

With this purpose in mind we present in this Letter large-scale exhaustive enumeration of microscopic states for on-lattice 3-D network models of glassy systems 2123 . By placing the system on a lattice, a procedure successfully employed for polymers [24,25, we can analyze in detail regions of configuration space containing many thousands of local minima, each minimum representing a topologically different network.

Our analysis shows that the local trap geometry can be characterized rather simply. The discussion deals with the connection between the geometry and dynamical features of glasses, including thermal metastability, domain formation and cooling behavior.

Model. The main structural feature of covalently bonded glasses as e.g. amorphous $\mathrm{SiO}_{2}$ close to the transition temperature is their topology. Accordingly, such systems are often modeled as random networks of building blocks [26] where links represent the covalent bonds.

Since we are interested in configurational changes which take place on time and length scales considerably larger than those appropriate for the vibrational degrees of freedom of the system, we can put our network on a grid with a lattice spacing $a$ of the order of the bond lenght. The harmonic vibrations cannot be described at this level of resolution. Furhermore, the energy minima of the discrete model and the corresponding positions of the atoms will be somewhat changed in comparison with the off-lattice network. However, overall geomerical features of the energy landscape as e.g. the distribution of accessible minima and barriers are not likely to be seriously affected.

We use cubic lattices with lattice constant $a=1$, periodic boundary conditions and a repeated cell of linear 
size $S=10$ and volume $V=S^{3}$. The number of atoms is $N_{A}$ and the density is $\rho=N_{A} / V$.

There is an arbitrariness in the choice of the elementary moves which define the dynamics on the energy landscape and hence its connectivity. We were guided by the simple physical consideration that a single move should involve a change of coordinates which is small. Each elementary move is therefore taken as a shift of the position of one atom to one of the six adjacent grid points. With this definition each state space configuration has $6 N_{A}$ neighbors in the landscape.

Our interaction potential is constructed such that a well defined crystalline ground state is present at a certain density. At the same time it allows for many other stable configurations with no crystalline structure. The potential vanishes except for nearest neighbors, which are atoms closer than $r=3.5$. The nearest neighbor interaction consists of a two-body and a three-body term. The radial dependence of the former has a hard-core for $r<1.6$, and equals $V_{2}(r)=(r-1.97)(r-3.5)^{2}(r-7)$, for $1.6<r<3.5$. This potential has a minimum at $r \approx 2.2$.

The three-body term $V_{3}(\theta)$ containing the angular dependence is infinite for angles below $40^{\circ}$. In the range $\left[40^{\circ}, 80^{\circ}\right]$ it is strongly repulsive and given by $V_{3}(\theta)=$ $-2.9 \times 10^{-7}(\theta-80)(\theta-180)^{2} \theta$. In the range $\left[80^{\circ}, 180^{\circ}\right]$ the potential has a shallow broad minimum close to $120^{\circ}$ and vanishes at the high end of the interval, as expressed by the form: $V_{3}(\theta)=-5.8 \times 10^{-8}(\theta-80)(\theta-180)^{2} \theta$. The optimal local coordination in the dense phase is the result of the interplay of $V_{2}(r)$ and $V_{3}(\theta)$, the former favoring high coordination (up to six neighbors in an octahedral arrangement), and the latter one favoring a trigonal planar environment. As a consequence, four-fold (tetrahedral) coordination is favored energetically, followed closely by five-fold coordination. Configurations connected by translations and/or rotations of the system and hence physically equivalent are identified and counted once.

Results. The parallel version of the lid-algorithm utilized for our exhaustive searches is detailed in Ref. [27]. The basic idea of how the method works is best explained by referring to the data shown in Fig. 1. For concreteness we shall focus on the cone-like structures pertaining to the 50 atom system. The algorithm starts at the reference configuration represented by the apex of the highest lying cone and visits all configurations which can be reached by a succession of elementary moves never exceeding an energy barrier $L$. The latter appears as the vertical distance from the apex along the cones' symmetry axis. The volume $\mathcal{V}(L)$ of a pocket of depth $L$ is then the number of configurations reachable below energy $L$. In the figure, the width of the cone at distance $L$ from the apex is proportional to the logarithm of $\mathcal{V}(L)$. As we see, the accessible volume is almost exponentially increasing with the barrier. At some value $L_{\max }$ of the barrier, which in the case of the highest pocket is $\approx 0.5 \mathrm{eV}$, the algorithm finds a state of energy lower than the lowest energy so far. This state is adopted as a new reference state, and the counting starts afresh. By iterating this procedure the algorithm generates a succession of cones located at progressively lower energies. Each of these is the graphical rendering of a state-space pocket. We emphasize that, while the counting within each pocket is exhaustive, the path joining one pocket to the next (symbolized by the lines connecting the cones) is just one amongst the many possible. The largest pockets contain $\approx 10^{5}-10^{6}$ states. A second quantity of importance is the local density of states $\mathcal{D}(E ; L)$, describing the energy distribution of the states in a pocket of depth $L$. For some representative instances $\ln \mathcal{D}$ is plotted versus $E$, where $0 \leq E \leq L$, in the same way as $\mathcal{V}(L)$ was plotted versus $L$. This results in the conical shapes enclosed in the dashed boxes of Fig. 1, which show that $\mathcal{D}(E ; L)$ is also close to an exponential function of its argument. A more detailed analysis shows that the curves have a slightly concave appearance in a semilog plot.

Figure 1 may convey the false impression that the pockets are devoid of internal structure. In reality, they all contain a multitude of local minima, each surrounded by its own basin. In fact, the number of accessible minima and their density also grow approximately exponentially as a function of $L$ and $E$ (for fixed $L$ ), respectively. Part of this rich structure is made visible in Fig. 2, where we consider one of the previously depicted pockets (deepest minimum for $N_{A}=45$ ) in more detail. The local density of states of a pocket (top curve) and six of its subpockets are displayed. We remark that each sub-pocket contains many local minima, and all the local densities of states have a similar, close to exponential appearance. We have also investigated the distribution in energy of the local minima in each pocket finding shapes similar to that of the local density of states, except for stronger flattening close to the lid energy.

In a harmonic approximation of the vibrational modes within a local minimum, one would expect a power-law form of the density of states. The present local densities of states, however, pertain to a rather different situation, i.e. the much larger length and time scales of excitations associated with changes in bonding topology. To check whether a power-law could nonetheless describe the data, we shift the abscissa with the energy of the lowest minimum in each valley and plot the data in a log-log plot. The inset of Fig. 2 shows two of the curves of the main panel, plotted in the fashion just described.

Finally, we considered the radial and angular distribution functions $G(r)$ and $P(\theta)$ for networks of different densities. In Fig. 3, we depict $P(\theta)$ averaged over the lowest minima for each density. The fact that the strain in the network increases with density is revealed by the increasing predominance of 'bad' angles.

The acquisition process for all of our data took of the order of one year of calculation, running in parallel on 8 
processors on a SGI Onyx computer.

Discussion. The local geometrical structure of each pocket can be described in terms of available volume, local density of states, and local density of minima. Even though these quantities vary somewhat from pocket to pocket, they nevertheless share important features. Approximately, the local density of states within a pocket of depth $L$ is $\mathcal{D}(E, L) \approx C(L) \exp \left(E / T_{g}\right)$. The parameter $1 / T_{g}$ characterizes the average slope of $\mathcal{D}$ in a semilog plot and the function $C(L)$ describes the effects of sidevalleys joining the main valley as the lid grows. Integrating $\mathcal{D}$ with respect to $E$ from $E=0$ up to $E=L$ yields $\mathcal{V}(L) \propto C(L) \exp \left(L / T_{g}\right)$. Since, as seen in Fig. 1, $\mathcal{V}(L)$ also grows almost exponentially as a function of $L$, we conclude that $C(L)$ must itself be an exponential or equal to a constant. In the latter case all the states added by increasing the lid would have energies very close to the lid itself, which in turn implies that the local minima are shallow or lacking altogether. In fact, however, deep sub-pockets frequently appear as the lid is increased. Since these side-pockets have fewer states than the main pocket, but the same overall shape with a lesser barrier to a deeper minimum, they are approximately scaled-down versions of their parent. Referring to Fig. 1 we note that this scaling property also seems to apply when moving one level up from the individual pockets to the larger scale structure of the whole landscape. This is because a high-lying pocket is by construction a subset of a lower-lying one and because all pockets within a certain energy range seem to have similar shapes independently of the energy of their lowest minimum. All in all, this is strongly suggestive of a self-similar structure in the energy landscape. Of course, to rigorously check self-similarity one should extend the analysis to several additional levels of nesting, a task which falls outside the scope of this investigation. From what we can glean so far concerning the largest energy scale, where we also would include very high-lying (possibly physically non-relevant) minima, there appears to be a weak trend to higher values of $T_{g}$ for the high-lying pockets. But since these narrow pockets usually appear to be relatively small compared to the rest of the system, their influence on the dynamical properties should not be very strong. Quite generally, the information gained from these exhaustive searches allows the construction or/and validation of mesoscopic models of complex system dynamics [28 30] which rely on assumed geometrical properties of state space. Since our pockets are metastable below the trapping temperature $T_{g}$ one can construct and use the connectivity matrix of small pockets $\left(<10^{4}\right.$ states $)$ to obtin direct insight in the low-temperature relaxation behavior [9].

For $T>T_{g}$, a hypothetical quasi-equilibrium distribution within the pocket would be very skewed in favor of states with energy close to the lid [9, 31, 32]. Banning unlikely kinetic mechanisms which would prevent the system from escaping the trap, this implies a loss of metastability at $T \approx T_{g}$, where the trap effectively disappears from the landscape. A purely exponential density of states gives a singularity in the specific heat when the temperature approaches $T_{g}$ from the low side. (The high side is irrelevant since the system has then left the pocket and new unchartered parts of state space must enter the calculation). Such a peak in $C_{V}$ is also observed experimentally at the glass transition temperature, and commonly associated with a configurational entropy [21,22]. The finite size of the pocket and corrections to the purely exponential behavior smoothen the transition of course.

The value of $T_{g}$ decreases with the system real space volume $V$ 23, 31. In the glass case, a simple free volume analysis 23 indicates that $T_{g} \propto 1 /\left(V\left(1-\rho V_{A}\right)\right)$, where $V_{A}$ is the volume/atom in a dense strain-free network and $\rho$ is the particle density. The fact that $T_{g}$ vanishes in the limit of infinite volume does not imply that trapping does not take place in large systems. Indeed, a large system will not be able to equilibrate internally at low temperatures, and the interpretation of $T_{g}(V)$ as a trapping temperature is then no longer appropriate. Instead one may envisage that a large system may form domains of strongly correlated atoms, similarly to the behavior observed in spin-glasses 33 35. The preceding analysis applied to these domains leads to the conclusion that the domain size is limited by an entropic mechanism: for a given temperature $T$ a domain can only be stable if $T_{g}\left(V_{e f f}\right) \leq T$. Domains can grow up to the size at which equality holds in the above relation.

If the system is annealed from high temperatures a slower cooling rate will arguably lead to a larger size $V_{\text {eff }}$ of locally equilibrated domains, and hence to a lower glass transition temperature, which agrees with the experimental behavior of glasses under cooling [21,22].

The basic condition for the above scenario, i.e. the approximate exponential growth of local densities of states in locally ergodic regions of the energy landscape, is fulfilled in a number of additional systems that show complex dynamics and glass-like behavior: the TSP [9], spin glasses [31], and lattice polymers [36. Thus, we suggest that a loss of metastability in spatially localized traps with exponential densities of states, i.e. mainly an entropic mechanism, is an important factor in the glass transition and in cooling rate dependent dynamic effects.

\section{Acknowledgments}

Funding was kindly provided by the DFG via SFB408 and by a block grant from Statens Naturvidenskabelige Forskningsråd.

[1] Spin glasses, K. H. Fischer and J. A. Hertz. Cambridge University Press,Cambridge, 1991. 
[2] R. Stephen Berry. Chem. Rev., 93:2379, 1993.

[3] K. D. Ball, R. S. Berry, R. E. Kunz, F.-Y. Li, A. Proykova, D. J. Wales Science 271:963, 1996.

[4] O. M. Becker, M. Karplus J. Chem. Phys., 106:1495, 1997.

[5] D. J. Wales, M. A. Miller, T. R. Walsh Nature, 394:758, 1998.

[6] C. M. Dobson, A. Sali and M. Karplus. Angew. Chem Int. Ed. Engl. 37:868, 1998.

[7] Landscape paradigms in Physics and Biology, Hans Frauenfelder, Alan R. Bishop, Angel Garcia, Alan Perelson, Peter Schuster, David Sherrington and Peter J. Swart Eds. Physica D 107, Nos. 2-4, 1996.

[8] R. Palmer. Adv. Phys. 31:669, 1982.

[9] P. Sibani, J. C. Schön, P. Salamon and J.-O. Andersson. Europhys. Lett., 22:479, 1993.

[10] T. Klotz and S. Kobe. Acta Phys. Slov. 44:347, 1994.

[11] T. Klotz, S. Schubert and K. Hoffmann. Europ. Phys.J. B 2:313, 1998.

[12] J. C. Schön, H. Putz and M. Jansen. J. Phys.:Cond. Matter., 8:143, 1996.

[13] A. Heuer. Phys. Rev. Lett., 78:4051, 1997

[14] T. Aihara Jr., Y. Kawazoe and T. Masumoto. J. NonCryst. Solids, 205-207:875, 1996

[15] C. Oligschleger and J. C. Schön. J. Phys. Cond. Matter, 9:1049, 1997

[16] C. Oligschleger. Phys. Rev. B, in press

[17] L. J. Lewis. Phys. Rev. B, 44:4245, 1991

[18] W. Kob and J.-L. Barrat. Phys. Rev. Lett., 78:4581, 1997 and with a comment by U. Müssel and H. Rieger, Phys. Rev. Lett., 81:930 (1998)

[19] T. A. Weber and F. H. Stillinger. Phys. Rev. B, 32:5402, 1985

[20] R. G. D. Valle and H. C. Andersen. J. Chem. Phys., 97:2682, 1992

[21] Physics of Amorphous Materials, S. R. Elliott. Longman Scientific \& Technical, Essex, 1990

[22] The Vitreous State, I. Gutzow and J. Schmelzer. Springer,Berlin, 1995

[23] J. C. Schön and Paolo Sibani. J. Phys. A: Math. Gen. 31:8167, 1998.

[24] K. Kremer and K. Binder. Comp. Phys. Rep., 7:259, 1988

[25] K. Binder, J. Baschnagel, W. Paul, H.P. Wittmann and M. Wolfgardt. Comp. Mater. Sci., 4:309, 1995

[26] The Physics of Amorphous Solids, R. Zallen. Wiley,New York, 1983

[27] P. Sibani, Ruud v.d. Pas and J. C. Schön. Comp. Phys. Comm., 116:17, 1999

[28] P. Sibani and K. H. Hoffmann. Phys. Rev. Lett., 63:2853, 1989

[29] J. P. Bouchaud, E. Vincent and J. Hammann. J. Phys. I France, 4:139, 1994

[30] C. Uhlig, K. H. Hoffmann and P. Sibani. Z. Phys. B, 96:409, 1995

[31] P. Sibani. Physica A, 258:249, 1998 and P. Sibani and P. Schriver Physical Review B, 49:6667, 1994

[32] J. C. Schön. J. Phys. A: Math. Gen. 30:2367, 1997

[33] D. S. Fisher and D. A. Huse, Phys. Rev. B 38:386, 1988 Physica A 229:259, 1996

[34] J. Kisker, L. Santen, M. Schreckenberg, and H. Rieger Phys. Rev. B 53:6418, 1996
[35] J.-O. Andersson and P. Sibani Physica A 229:259, 1996

[36] J. C. Schön. Habilitation Thesis., Univ. Bonn, 1997 


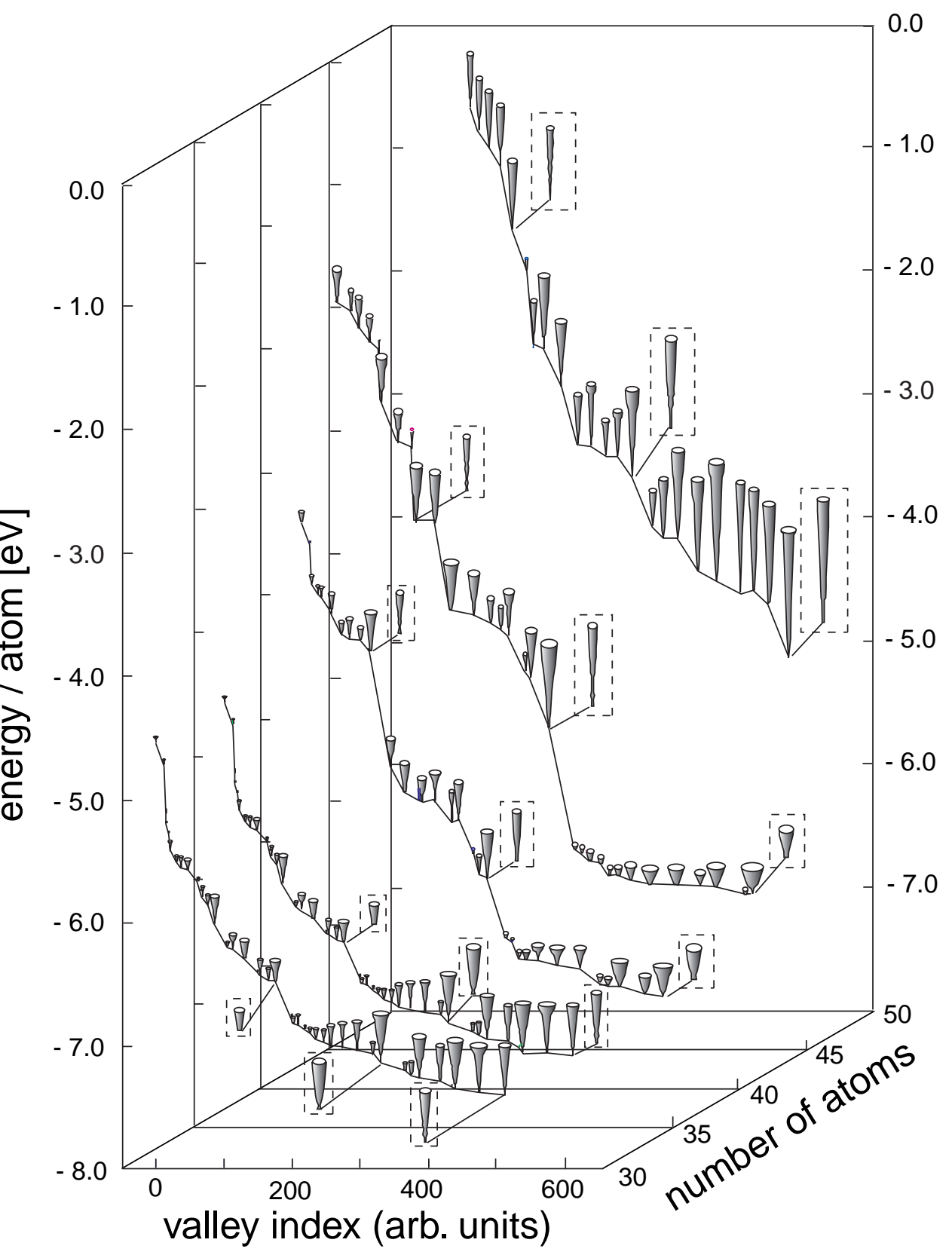

FIG. 1. A birds-eye view of the energy landscape of the glass model, where pockets appear as cone-like structures. The width of the cones at energy $L$ relative to the position of the apex is proportional to the logarithm of the locally accessible state-space volume $\mathcal{V}(L)$. The insets use the same graphical rendering but with the cone-width at energy $E$ proportional to the logarithm of $\mathcal{D}\left(E ; L_{\max }\right)$, the local density of states available in the selected pockets. 


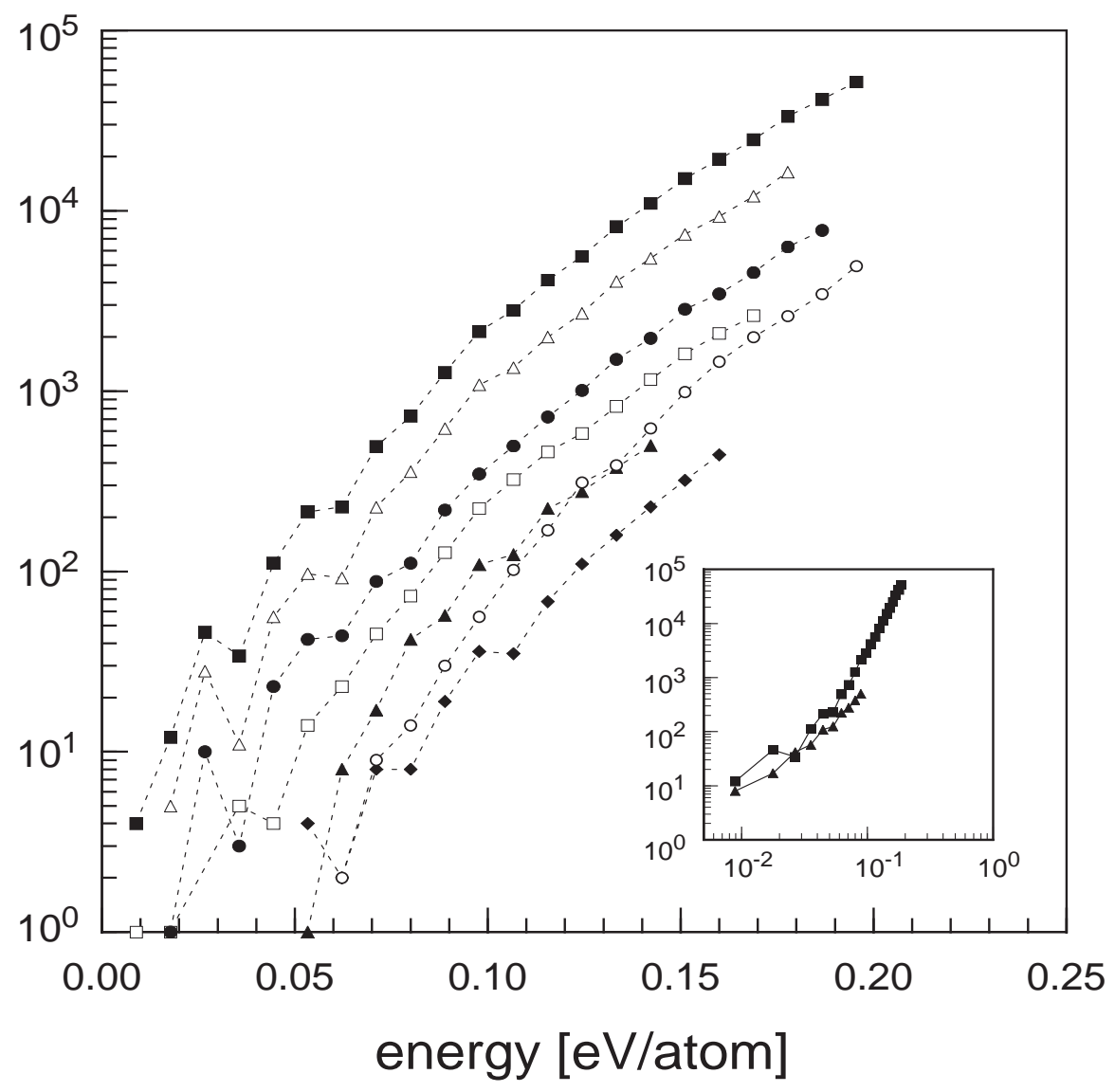

FIG. 2. The local density of states of several nested sub-pockets of a system with 45 atoms. The top curve (black squares) describes the local density of all states in the full pocket up to an energy of $\approx 0.22 \mathrm{eV} /$ atom. Each other curve describes the local density of states of a sub-pocket merging into the main valley approximately at the energy where the curve ends, within the accuraqcy given by the binning of the energy axis. E. g., the second curve (empty triangles) corresponds to a subpocket merging at $\approx 0.19 \mathrm{eV} /$ atom. Note that the growth of the local densities of states of the sub-pockets closely parallels the one of the main pocket. The inset shows the same data as the top and bottom curves of the main panel, plotted on log-log scales and with the abscissa values shifted, such that the lowest energy in the appropriate (sub)valley is located at the origin. 


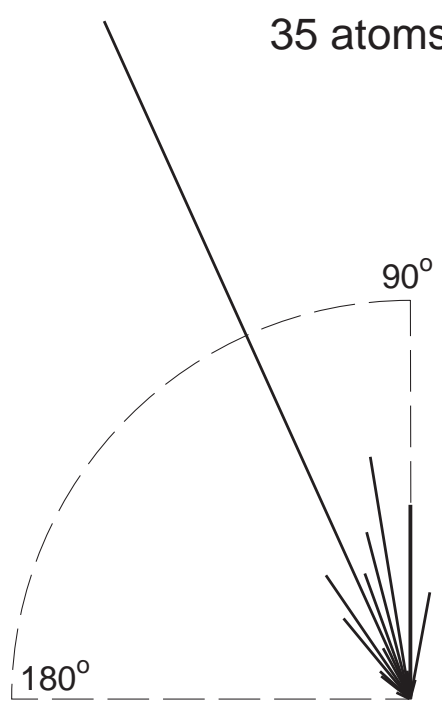

45 atoms

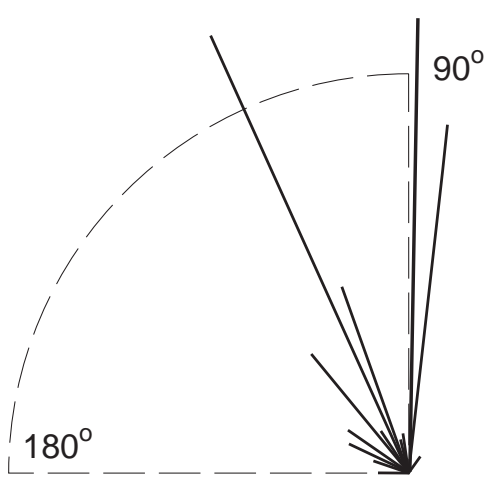

40 atoms
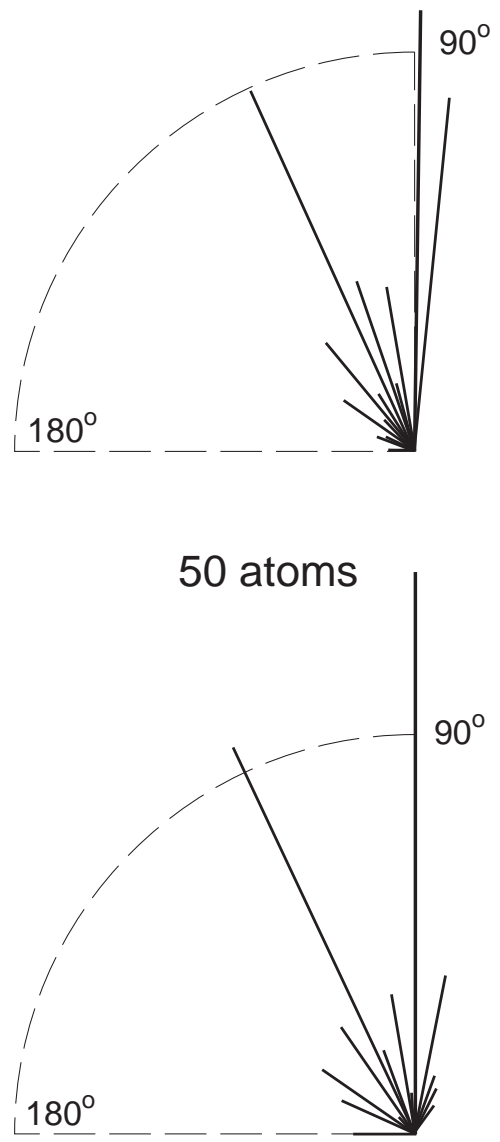

FIG. 3. The length of each ray is proportional to the frequency of the corresponding bond angle in the configurations of the deepest energy minima of the three lowest pockets for systems of 35, 40, 45, 50 atoms, respectively. The 30 atom system (not shown) is similar to the 35 atom one, exhibiting even more (ideal) tetrahedral surroundings. The preferred bond angle is close to $110^{\circ}$, due to the interplay of the two-bodyand the three-body-terms in the interaction potential. Note the increasing predominance of energetically unfavorable angles as the atom density increases. 